\title{
Análise espacial dos homicídios intencionais de mulheres*
}

\author{
Spatial analysis of female intentional homicides \\ Análisis espacial de los homicidios intencionales de mujeres
}

Como citar este artigo:

Barros SC, Oliveira CM, Silva APSC, Melo MFO, Pimentel DR, Bonfim CV. Spatial analysis of female intentional homicides. Rev Esc Enferm USP. 2021;55:e03770. https://doi.org/10.1590/S1980-220X2020037303770

\section{Sheyla Carvalho de Barros ${ }^{1}$ \\ Conceição Maria de Oliveira ${ }^{2}$ \\ Amanda Priscila de Santana Cabral Silva ${ }^{3}$ \\ D Marcella Fernandes de Oliveira Melo ${ }^{4}$ \\ Dayane da Rocha Pimentel ${ }^{5}$ \\ Cristine Vieira do Bonfim ${ }^{6}$}

* Extraído da dissertação "Homicídios e violência prévia contra mulher em Pernambuco", Programa de Pós-Graduação em Saúde Coletiva Universidade Federal de Pernambuco, 2019.

${ }^{1}$ Universidade Federal de Pernambuco, Programa de Pós-Graduação em Saúde Coletiva, Recife, PE, Brasil.

${ }^{2}$ Centro Universitário Mauricio de Nassau, Recife, PE, Brasil.

${ }^{3}$ Universidade Federal de Pernambuco, Centro Acadêmico de Vitória, Vitória de Santo Antão, PE, Brasil.

${ }^{4}$ Universidade Federal de Pernambuco, Recife, PE, Brasil.

${ }^{5}$ Instituto Aggeu Magalhães,

Programa de Pós-graduação em Saúde Pública, Recife, PE, Brasil.

${ }^{6}$ Fundação Joaquim Nabuco, Diretoria de Pesquisas Sociais, Recife, PE, Brasil.

\section{ABSTRACT}

Objective: To analyze the characteristics and spatial distribution of female intentional homicides, according to the regional health offices, in a state in the Northeast Region of Brazil. Method: This is an ecological study that had the municipality and regional health offices as units of analysis and used data from the Brazilian Mortality Information System. Mortality rates were calculated and smoothed by the local empirical Bayesian method. To identify the existence of spatial autocorrelation, the Moran's index was used. Results: A total of 1,144 homicides were reported, with an average rate of 6.2/100,000 women. For all regional health offices, the 10-39 age group, black race/skin color, and single marital status prevailed. The homicides took place in public spaces. The days of the week with the highest records were Saturday and Sunday and the months of the year December, January, February, and June. The global Moran's index was 0.6 ( $p=0.01$ ). Clusters of municipalities with high homicide rates were identified in regional health offices III and IV. Conclusions: The homicide victims were young, black, and single. The spatial analysis found critical areas of homicide occurrence, which are a priority for actions to prevent violence against women.

\section{DESCRIPTORS}

Homicide; Violence Against Women; Information Systems; Vital Statistics; Spatial Analysis. 


\section{INTRODUÇÃO}

A violência tem sido reconhecida como um dos principais problemas de saúde pública global. Cerca de 1,4 milhão de pessoas morrem anualmente devido à violência e o homicídio representa a forma mais extrema dessa violência ${ }^{(1-2)}$. Define-se homicídio intencional como "a morte ilegal propositalmente infligida a uma pessoa por outra"(1). No ano de 2012, o homicídio intencional causou cerca de meio milhão de mortes no mundo. Os homens cometem aproximadamente $95 \%$ dos homicídios e constituem 79\% das vítimas; no entanto, quando o homicídio está associado à violência conjugal ou intrafamiliar, afeta desproporcionalmente mais mulheres ${ }^{(3-4)}$.

Todos os anos, parceiros íntimos ou familiares cometem quase 64.000 homicídios intencionais. Do total de mulheres assassinadas, $47 \%$ são vítimas de parceiros íntimos ou familiares, enquanto apenas $6 \%$ dos homens, vítimas de homicídio, têm o parceiro íntimo ou familiar como o autor da violência ${ }^{(1)}$. Os homicídios de mulheres por razões de gênero são considerados feminicídios ${ }^{(5)}$.

A violência contra a mulher é um fenômeno que ainda persiste nas sociedades atuais e manifesta-se de diferentes maneiras (físicas, sexuais e psicológicas) e níveis de gravidade. Na maioria das vezes, ocorre dentro do contexto familiar, mais especificamente por parceiro íntimo, manifestando-se dentro de um relacionamento abusivo, com significativo impacto econômico, sociofamiliar e graves consequências para a saúde física e mental das vítimas. Muitas vezes o ponto final da violência contra a mulher é o homicídio ${ }^{(4)}$.

No Brasil, as mulheres expostas à violência têm um risco de mortalidade oito vezes maior que o da população geral de mulheres e estima-se que 100 mulheres morrem semanalmente como consequência direta ou indireta da exposição à violência $^{(6)}$. Houve mais de 100.000 homicídios de mulheres entre 1980 e 2014, com uma taxa de 5,13 óbitos por 100.000 mulheres ${ }^{(7)}$. No estado de Pernambuco, entre 2006 e 2016, a taxa média de homicídios na população feminina foi de $5,7 / 100.000$ mulheres $^{(8)}$.

As altas taxas de homicídios de mulheres no país e no mundo evidenciam a magnitude da violência de gênero na sua forma mais extrema. As características das vítimas e dos óbitos são informações que podem ser usadas para formulação das políticas públicas de prevenção da violência e redução de mortes. Outra ferramenta que pode ser utilizada pela área da saúde para estudar a violência é a análise espacial, que possibilita observar padrões de distribuição e identificar as áreas de risco para mortalidade por homicídios ${ }^{(9)}$. O objetivo deste estudo foi analisar as características e a distribuição espacial dos homicídios intencionais de mulheres, de acordo com as regionais de saúde, em um estado da Região Nordeste do Brasil.

\section{MÉTODO}

\section{DESENHO DO ESTUDO}

Estudo ecológico que considerou como unidade espacial de análise os municípios do estado de Pernambuco e as regiões de saúde. O estado divide-se em 184 municípios e um distrito (Arquipélago de Fernando de Noronha), distribuídos por cinco mesorregiões e 12 regionais de saúde (RS). O distrito de Fernando de Noronha não foi incluído na análise espacial pela não existência de municípios limítrofes, porém não havia registro de homicídios intencionais de mulheres nesse distrito.

\section{População}

Foram incluídos todos os homicídios intencionais de mulheres, residentes no estado e que tinham 10 anos ou mais de idade, codificados como "causas externas de morbidade e de mortalidade” (códigos X85 a Y09 - Agressões), da Classificação Estatística Internacional de Doenças e Problemas Relacionados à Saúde (CID-10), registrados no Sistema de Informações sobre Mortalidade (SIM) no período de 2012 a 2016.

\section{Coleta De Dados}

No mês de novembro de 2018 foi obtido o banco de dados do SIM, referente aos homicídios intencionais de mulheres, junto à Secretaria de Saúde do Estado de Pernambuco. Utilizaram-se as projeções da população do Instituto Brasileiro de Geografia e Estatística (IBGE); a base digital também é proveniente do IBGE, com sistema de referência Sirgas 2000 e sistema de coordenadas geográficas.

\section{ANÁlISE E TRATAMENTO DOS DADOS}

Para caracterização dos homicídios em mulheres foram estudadas as variáveis: faixa etária (10 a 39 anos, 40 e mais); raça/cor (não negra = branca, negra = pretas + pardas); situação conjugal (solteira, casada/união estável, viúva, divorciada); local de ocorrência do óbito (hospital/serviço de saúde, domicilio, via pública, outros); mês e dia da semana de ocorrência do óbito; e causa básica do óbito (disparo de arma de fogo; objeto cortante ou penetrante; objeto contundente; enforcamento, estrangulamento, sufocação; e outros meios).

Foram calculadas as taxas médias de mortalidade por 100 mil, dividindo-se a média de homicídios de mulheres pela média da população de mulheres residentes no estado de Pernambuco, com 10 anos ou mais de idade, no período estudado. A opção por utilizar a taxa média teve o objetivo de evitar o viés decorrente dos pequenos números.

As taxas médias de mortalidade foram ajustadas pelo método bayesiano empírico local, com o propósito de minimizar os efeitos das flutuações decorrentes do pequeno número de óbitos em alguma das unidades territoriais. Foi utilizado o índice de Moran global para verificação da autocorrelação espacial das taxas. Esse índice varia de $-1 \mathrm{a}+1$, onde zero indica ausência de autocorrelação e valores mais próximos de 1 indicam a existência de autocorrelação positiva ou negativa. Quanto mais próximo de 1, maior a semelhança entre os municípios vizinhos em relação à mortalidade.

Para a delimitação das áreas críticas e de transição, bem como a identificação de clusters, empregou-se o índice de Moran local (Local Indicators of Spatial Association - LISA). Foram construídos o Box Map e o Moran Map das taxas de mortalidade. Os clusters foram classificados em quatro 
quadrantes: Q1 (valores positivos, médias positivas) e Q2 (valores negativos, médias negativas); Q3 (valores positivos, médias negativas) e Q4 (valores negativos, médias positivas). No Box Map, os clusters são apresentados independentemente da significância estatística; e no Moran Map, permanecem os clusters com significância ( $\mathrm{p}$-valor $<0,05$ ). Áreas de alto risco corresponderam àquelas em que o município apresenta alta taxa de mortalidade e os seus vizinhos também têm altas taxas, classificadas no Q1. Foi realizada a distribuição geográfica do Índice de Desenvolvimento Humano (IDH) por município, no estado de Pernambuco, que se baseia em dimensões (educação, saúde e renda) para aferir o desenvolvimento de uma localidade ${ }^{(10)}$. O programa TerraView (versão 4.2) foi utilizado para cálculo e análise dos indicadores de autocorrelação espacial e o programa QGIS (versão 2.18) para apresentação gráfica.

\section{Aspectos ÉTIICOS}

A pesquisa respeitou as normas e diretrizes éticas da Resolução no 466/2012, do Conselho Nacional de Saúde e foi aprovada pelo Comitê de Ética em Pesquisa da Universidade Federal de Pernambuco sob o parecer no 2.986.711, de 29 de outubro de 2018. Contou com a anuência da Secretaria de Saúde do Estado de Pernambuco, para cessão do banco de dados do SIM.

\section{RESULTADOS}

No período estudado, foram registrados 1.144 homicídios intencionais de mulheres. Desses, 503 (43,9\%) concentraram-se na I RS. A idade das vítimas variou de 10 a 97, com média de 24 anos (desvio padrão = 15,3 anos). Para todas as RS prevaleceram a faixa etária de 10 a 39 anos de idade, a raça/cor negra e a situação conjugal solteira. Em relação ao local de ocorrência, em seis RS e no estado de
Pernambuco, homicídios ocorreram predominantemente em via pública. A maior parte dos homicídios ocorreu no final de semana (sábado e domingo) em cinco RS e em Pernambuco. Os meses do ano com maiores registros de homicídios foram dezembro $(9,1 \%)$, janeiro $(8,8 \%)$, fevereiro $(8,8 \%)$ e junho $(8,8 \%)$ (Tabela 1$)$.

O disparo de arma de fogo foi a principal causa em sete RS e no estado de Pernambuco. Destaca-se que na I RS, 332 $(66,0 \%)$ homicídios foram por disparo de arma de fogo. Para as RS VII, VIII e IX, a maior frequência foi objeto cortante ou penetrante (Tabela 2 ).

A taxa média de mortalidade foi de 6,2, variando de 1,2 a 17,0 (Figura 1A). Entre as RS VII e VI, a taxa variou de 2,3 a 7,0 , respectivamente. $\mathrm{O}$ município com menor taxa (1,2/100.000 mulheres) foi Santa Maria da Boa Vista, situado na VIII RS. O município com taxa mais elevada (17,1/100.000 mulheres) foi Camutanga, situado na XII RS.

A Figura $1 \mathrm{~B}$ apresenta as taxas suavizadas pelo estimador bayesiano empírico local e 25\% estão distribuídos pelas I, II, III, IV, V, VI, IX e XII RS, sendo a maior concentração na IV RS.

$\mathrm{O}$ índice de Moran global das taxas suavizadas foi de $0,6(p=0,01)$. No diagrama de espalhamento de Moran (Box Map), os municípios Q1 situaram-se principalmente na III, IV, V, VI e XII RS (Figura 1C). O Moran Map foi utilizado para identificação dos clusters de municípios com autocorrelação espacial estaticamente significante de 5\%. Identificaram-se quatro conglomerados e um município crítico (Q1), quatro conglomerados de baixo nível (Q2) e um município em transição (Q3). Os clusters (áreas Q1) estão localizados nas RS: I (dois municípios), III (oito municípios), IV (oito municípios), XII (dois municípios) e VI (três municípios, sendo um conglomerado e um município isolado) (Figura 1D). Os municípios que estão nas áreas Q1 possuem taxas de IDH nos níveis baixo e médio (Figura 1E).

Tabela 1 - Caracterização dos homicídios de mulheres, segundo regionais de saúde - Pernambuco, Brasil, 2012-2016.

\begin{tabular}{|c|c|c|c|c|c|c|c|c|c|c|c|c|c|c|c|c|c|c|c|c|c|c|c|c|c|c|}
\hline \multirow{2}{*}{ Regionais de Saúde } & \multicolumn{2}{|c|}{ I } & \multicolumn{2}{|c|}{ II } & \multicolumn{2}{|c|}{ III } & \multicolumn{2}{|c|}{ IV } & \multicolumn{2}{|c|}{ V } & \multicolumn{2}{|c|}{ VI } & \multicolumn{2}{|c|}{ VII } & \multicolumn{2}{|c|}{ VIII } & \multicolumn{2}{|c|}{ IX } & \multicolumn{2}{|c|}{$\mathrm{X}$} & \multicolumn{2}{|c|}{$\mathbf{X I}$} & \multicolumn{2}{|c|}{ XII } & \multicolumn{2}{|c|}{ PE } \\
\hline & $\mathbf{n}$ & $\%$ & $\mathbf{n}$ & $\%$ & $\mathbf{n}$ & $\%$ & $\mathbf{n}$ & $\%$ & $\mathbf{n}$ & $\%$ & n & $\%$ & $\mathbf{n}$ & $\%$ & $\mathbf{n}$ & $\%$ & $\mathbf{n}$ & $\%$ & $\mathbf{n}$ & $\%$ & $\mathbf{n}$ & $\%$ & $\mathbf{n}$ & $\%$ & $\mathbf{n}$ & $\%$ \\
\hline \multicolumn{27}{|l|}{ Faixa etária } \\
\hline 10 a 39 & 394 & 78,3 & 45 & 71,4 & 70 & 83,3 & 132 & 72,5 & 45 & 70,3 & 36 & 61,0 & 4 & 57,1 & 38 & 74,5 & 32 & 76,2 & 14 & 82,4 & 22 & 84,6 & 34 & 73,9 & 866 & 75,7 \\
\hline 40 e mais & 109 & 21,7 & 18 & 28,6 & 14 & 16,7 & 50 & 27,5 & 19 & 29,7 & 23 & 39,0 & 3 & 42,9 & 13 & 25,5 & 10 & 23,8 & 3 & 17,6 & 4 & 15,4 & 12 & 26,1 & 278 & 24,3 \\
\hline \multicolumn{27}{|l|}{ Raça/cor } \\
\hline Não negra & 69 & 13,9 & 9 & 14,8 & 10 & 12,2 & 20 & 11,0 & 5 & 8,2 & 5 & 8,5 & - & - & 4 & 8,0 & 4 & 9,8 & 3 & 17,6 & 1 & 3,8 & 4 & 8,9 & 134 & 11,9 \\
\hline Negra & 427 & 86,1 & 52 & 85,2 & 72 & 87,8 & 162 & 89,0 & 56 & 91,8 & 54 & 91,5 & 7 & 100,0 & 46 & 92,0 & 37 & 90,2 & 14 & 82,4 & 25 & 96,2 & 41 & 91,1 & 994 & 88,1 \\
\hline \multicolumn{27}{|l|}{ Situação conjugal } \\
\hline Solteira & 417 & 90,3 & 50 & 80,6 & 65 & 85,5 & 108 & 78,3 & 32 & 74,4 & 27 & 67,5 & 6 & 85,7 & 38 & 76,0 & 29 & 74,4 & 8 & 61,5 & 14 & 66,7 & 37 & 86,0 & 832 & 82,1 \\
\hline Casada/união estável & 45 & 9,7 & 10 & 16,1 & 6 & 7,9 & 20 & 14,5 & 10 & 23,3 & 10 & 25,0 & 1 & 14,3 & 10 & 20,0 & 6 & 15,4 & 1 & 7,7 & 4 & 19,0 & 2 & 4,7 & 125 & 12,3 \\
\hline Viúva & - & - & 1 & 1,6 & 3 & 3,9 & 7 & 5,1 & 1 & 2,3 & 2 & 5,0 & - & - & - & - & 2 & 5,1 & - & - & 2 & 9,5 & 2 & 4,7 & 31 & 3,1 \\
\hline Divorciada & - & - & 1 & 1,6 & 2 & 2,6 & 3 & 2,2 & - & - & 1 & 2,5 & - & - & 2 & 4,0 & 2 & 5,1 & 4 & 30,8 & 1 & 4,8 & 2 & 4,7 & 26 & 2,6 \\
\hline
\end{tabular}




\section{...continuação}

\begin{tabular}{|c|c|c|c|c|c|c|c|c|c|c|c|c|c|c|c|c|c|c|c|c|c|c|c|c|c|c|}
\hline \multirow{2}{*}{ Regionais de Saúde } & \multicolumn{2}{|c|}{ I } & \multicolumn{2}{|c|}{ II } & \multicolumn{2}{|c|}{ III } & \multicolumn{2}{|c|}{ IV } & \multicolumn{2}{|c|}{ V } & \multicolumn{2}{|c|}{ VI } & \multicolumn{2}{|c|}{ VII } & \multicolumn{2}{|c|}{ VIII } & \multicolumn{2}{|c|}{ IX } & \multicolumn{2}{|c|}{$X$} & \multicolumn{2}{|c|}{$\mathbf{X I}$} & \multicolumn{2}{|c|}{ XII } & \multicolumn{2}{|c|}{ PE } \\
\hline & $\mathbf{n}$ & $\%$ & $\mathbf{n}$ & $\%$ & $\mathbf{n}$ & $\%$ & $\mathbf{n}$ & $\%$ & $\mathbf{n}$ & $\%$ & $\mathbf{n}$ & $\%$ & $\mathbf{n}$ & $\%$ & $\mathbf{n}$ & $\%$ & $\mathbf{n}$ & $\%$ & $\mathbf{n}$ & $\%$ & $\mathbf{n}$ & $\%$ & $\mathbf{n}$ & $\%$ & $\mathbf{n}$ & $\%$ \\
\hline
\end{tabular}

Local de ocorrência

$\begin{array}{llllllllllllllllllllllllllll}\text { Hospital/Serviço de saúde } & 94 & 22,1 & 8 & 12,9 & 19 & 22,6 & 25 & 13,8 & 6 & 9,4 & 11 & 19,0 & - & - & 8 & 15,7 & 6 & 14,3 & 4 & 23,5 & 2 & 7,7 & 9 & 20,0 & 266 & 23,4\end{array}$

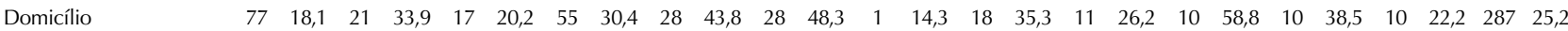
$\begin{array}{llllllllllllllllllllllllllll}\text { Via pública } & 225 & 52,8 & 28 & 45,2 & 37 & 44,0 & 87 & 48,1 & 24 & 37,5 & 12 & 20,7 & 1 & 14,3 & 11 & 21,6 & 6 & 14,3 & 1 & 5,9 & 11 & 42,3 & 21 & 46,7 & 464 & 40,8\end{array}$

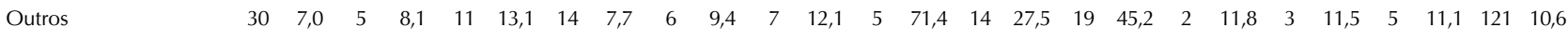

Mês da ocorrência

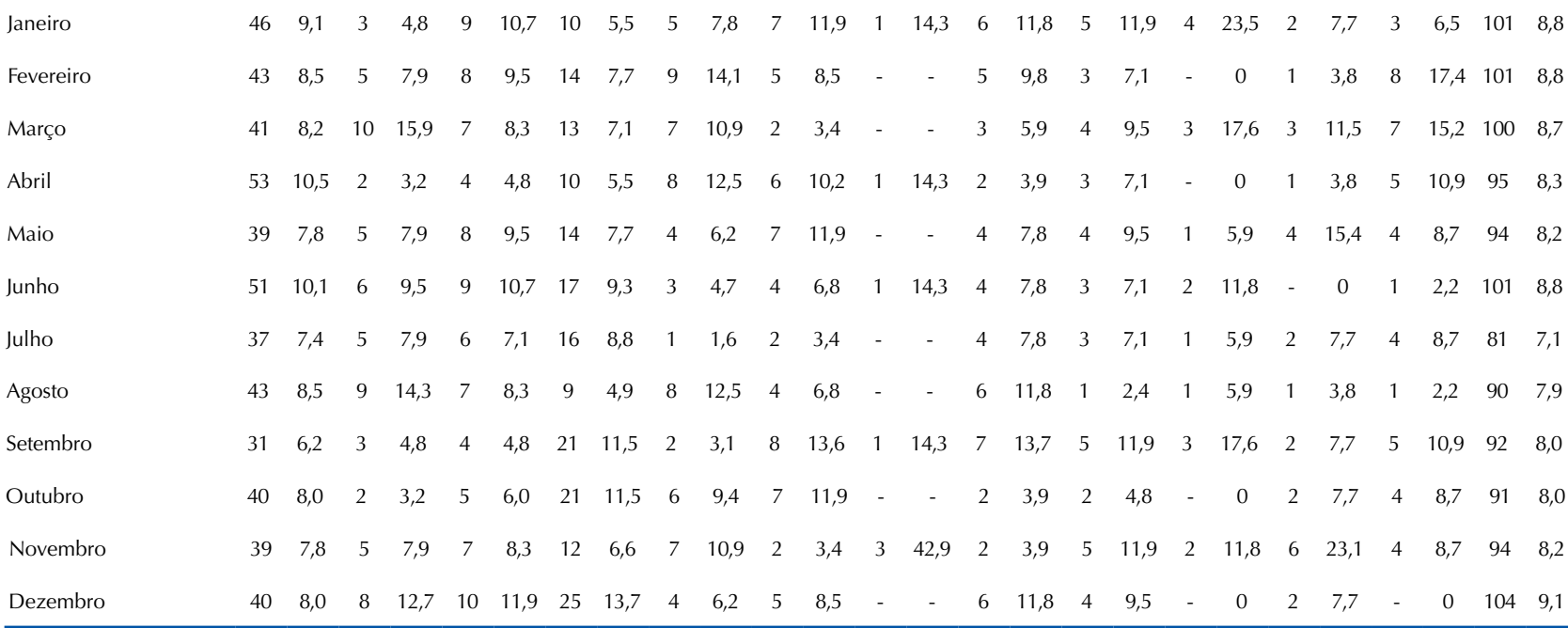

Dia da semana

Segunda

Terça

$\begin{array}{lllllllllllllllllllllllllll}78 & 15,5 & 10 & 15,9 & 12 & 14,3 & 33 & 18,1 & 7 & 10,9 & 10 & 16,9 & - & - & 7 & 13,7 & 5 & 11,9 & 4 & 23,5 & 4 & 15,4 & 5 & 10,9 & 175 & 15,3\end{array}$

Quarta

$\begin{array}{llllllllllllllllllllllllll}56 & 11,1 & 17 & 27,0 & 4 & 4,8 & 28 & 15,4 & 9 & 14,1 & 10 & 16,9 & 1 & 14,3 & 3 & 5,9 & 5 & 11,9 & 1 & 5,9 & 5 & 19,2 & 4 & 8,7 & 143 & 12,5\end{array}$

Quinta

$\begin{array}{llllllllllllllllllllllllll}65 & 12,9 & 9 & 14,3 & 9 & 10,7 & 17 & 9,3 & 8 & 12,5 & 6 & 10,2 & 0 & 0 & 6 & 11,8 & 8 & 19 & 2 & 11,8 & 5 & 19,2 & 8 & 17,4 & 143 & 12,5\end{array}$

Sexta

$\begin{array}{lllllllllllllllllllllllllllll}52 & 10,4 & 6 & 9,5 & 9 & 10,7 & 23 & 12,6 & 9 & 14,1 & 5 & 8,5 & 0 & 0 & 2 & 3,9 & 1 & 2,4 & 1 & 5,9 & 1 & 3,8 & 7 & 15,2 & 116 & 10,1\end{array}$

Sábado

$\begin{array}{lllllllllllllllllllllllllll}72 & 14,3 & 6 & 9,5 & 11 & 13,1 & 27 & 14,8 & 8 & 12,5 & 10 & 16,9 & 1 & 14,3 & 6 & 11,8 & 7 & 16,7 & 1 & 5,9 & 2 & 7,7 & 8 & 17,4 & 159 & 13,9\end{array}$

$\begin{array}{lllllllllllllllllllllllllll}88 & 17,5 & 6 & 9,5 & 18 & 21,4 & 27 & 14,8 & 11 & 17,2 & 5 & 8,5 & 2 & 28,6 & 15 & 29,4 & 3 & 7,1 & 4 & 23,5 & 5 & 19,2 & 4 & 8,7 & 188 & 16,4\end{array}$

Domingo

$\begin{array}{lllllllllllllllllllllllllll}92 & 18,3 & 9 & 14,3 & 21 & 25,0 & 27 & 14,8 & 12 & 18,8 & 13 & 22 & 3 & 42,9 & 12 & 23,5 & 13 & 31 & 4 & 23,5 & 4 & 15,4 & 10 & 21,7 & 220 & 19,2\end{array}$

Notas: Ignorados/em branco: raça/cor - 16 (1,39\%), situação conjugal - 130 (11,36\%), local de ocorrência - 06 (0,52\%)

Tabela 2 - Caracterização dos homicídios de mulheres por causa básica do óbito, segundo regionais de saúde - Pernambuco, Brasil, 2012-2016.

\begin{tabular}{|c|c|c|c|c|c|c|c|c|c|c|c|c|c|c|c|c|c|c|c|c|c|c|c|c|c|c|}
\hline \multirow{2}{*}{ Regionais de Saúde } & \multicolumn{2}{|c|}{ I } & \multicolumn{2}{|c|}{ II } & \multicolumn{2}{|c|}{ III } & \multicolumn{2}{|c|}{ IV } & \multicolumn{2}{|c|}{ V } & \multicolumn{2}{|c|}{ VI } & \multicolumn{2}{|c|}{ VII } & \multicolumn{2}{|c|}{ VIII } & \multicolumn{2}{|c|}{ IX } & \multicolumn{2}{|c|}{$\mathrm{X}$} & \multicolumn{2}{|c|}{$\mathbf{X I}$} & \multicolumn{2}{|c|}{ XII } & \multicolumn{2}{|c|}{ PE } \\
\hline & $\mathbf{n}$ & $\%$ & $\mathbf{n}$ & $\%$ & $\mathbf{n}$ & $\%$ & $\mathbf{n}$ & $\%$ & $\mathbf{N}$ & $\%$ & $\mathbf{n}$ & $\%$ & $\mathbf{n}$ & $\%$ & $\mathbf{n}$ & $\%$ & $\mathbf{n}$ & $\%$ & $\mathbf{n}$ & $\%$ & $\mathbf{n}$ & $\%$ & $\mathbf{n}$ & $\%$ & $\mathbf{n}$ & $\%$ \\
\hline \multicolumn{27}{|l|}{ Causa básica do óbito } \\
\hline $\begin{array}{l}\text { Disparo de arma } \\
\text { de fogo }\end{array}$ & 332 & 66,0 & 33 & 52,4 & 55 & 65,5 & 101 & 55,5 & 25 & 39,1 & 25 & 42,4 & 3 & 42,9 & 17 & 33,3 & 15 & 35,7 & 7 & 41,2 & 15 & 57,7 & 25 & 54,3 & 654 & 57,2 \\
\hline $\begin{array}{l}\text { Objeto cortante ou } \\
\text { penetrante }\end{array}$ & 98 & 19,5 & 22 & 34,9 & 20 & 23,8 & 51 & 28,0 & 22 & 34,4 & 22 & 37,3 & 4 & 57,1 & 22 & 43,1 & 17 & 40,5 & 7 & 41,2 & 6 & 23,1 & 16 & 34,8 & 307 & 26,8 \\
\hline Objeto contundente & 50 & 9,9 & 4 & 6,3 & 6 & 7,1 & 21 & 11,5 & 8 & 12,5 & 9 & 15,3 & - & - & 8 & 15,7 & 8 & 19,0 & 2 & 11,8 & 4 & 15,4 & 3 & 6,5 & 123 & 10,8 \\
\hline $\begin{array}{l}\text { Enforcamento, } \\
\text { estrangulamento, } \\
\text { sufocação }\end{array}$ & 20 & 4,0 & 3 & 4,8 & 3 & 3,6 & 5 & 2,7 & 6 & 9,4 & 2 & 3,4 & - & - & 3 & 5,9 & - & - & 1 & 5,9 & - & - & 2 & 4,3 & 45 & 3,9 \\
\hline Outros meios & 3 & 0,6 & 1 & 1,6 & 0 & 0,0 & 4 & 2,2 & 3 & 4,7 & 1 & 1,7 & - & - & 1 & 2,0 & 2 & 4,8 & - & - & 1 & 3,8 & - & - & 15 & 1,3 \\
\hline
\end{tabular}




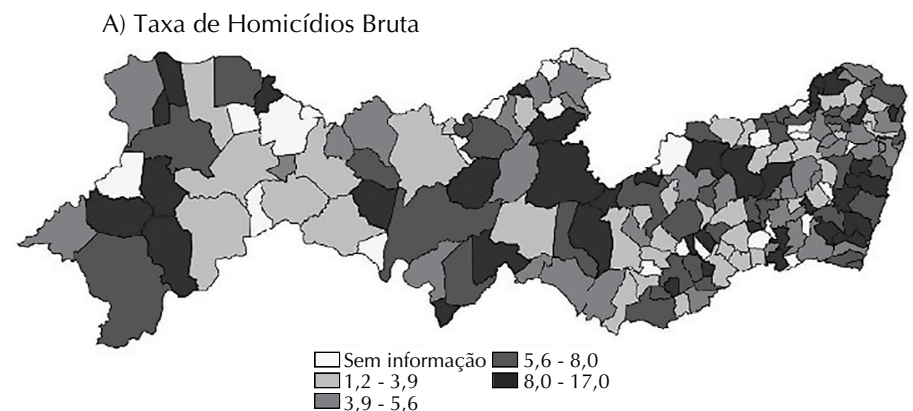

B) Bayesiano Empírico Local

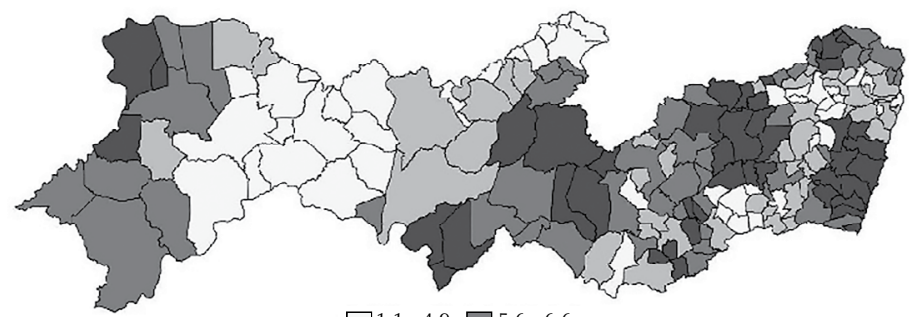

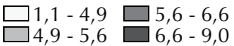

C) Box Map

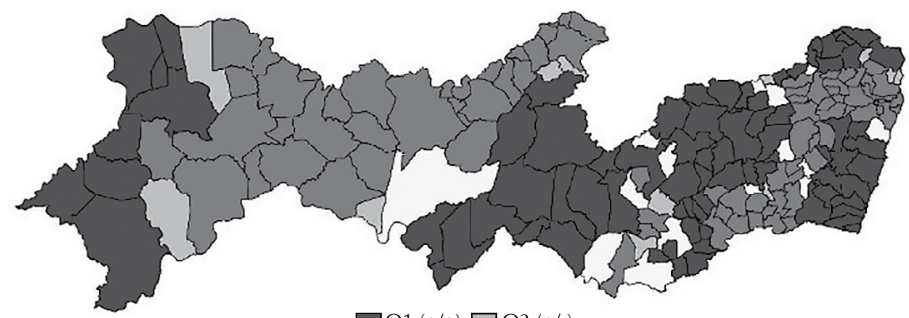

$\square \mathrm{Q} 1(+/+)$
$\square \mathrm{Q} 2(-/-)$
$\square$ Q $4(+/-)$

D) Moran Map

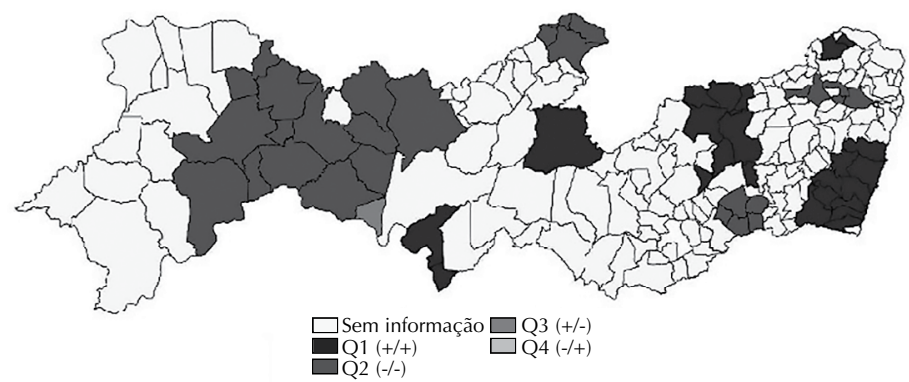

E) IDH-M
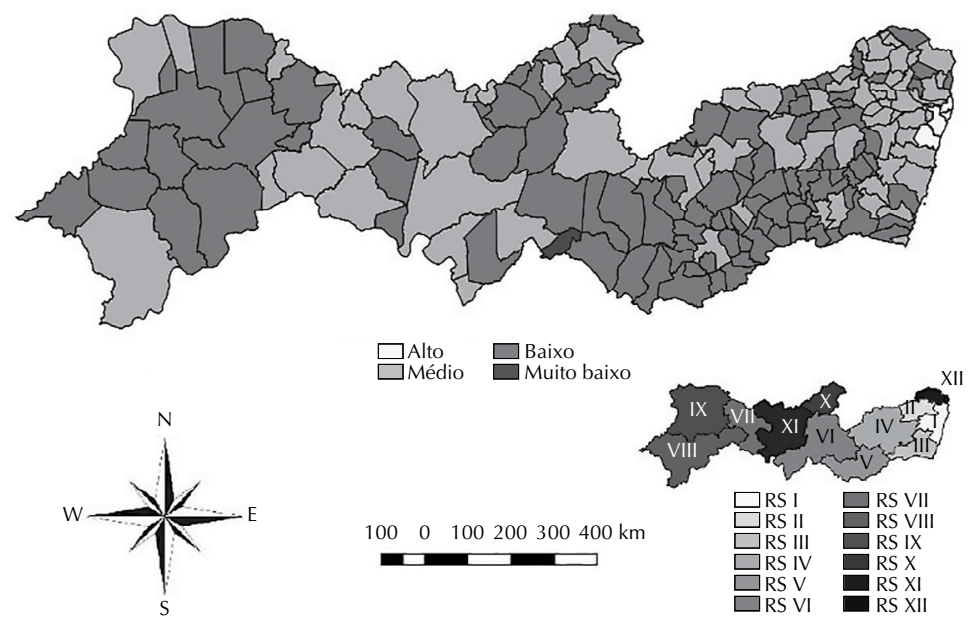

Figura 1 - Taxa média bruta (A), taxa média estimada pelo método bayesiano empírico local (B), distribuição espacial das taxas médias de homicídios - Box Map (C), áreas críticas para os homicídios, segundo o espalhamento de Moran - Moran Map (D) dos homicídios de mulheres (E), Índice de Desenvolvimento Humano por município - Pernambuco, Brasil, 2012-2016. 


\section{DISCUSSÃO}

Os resultados deste estudo são similares a outros encontrados na literatura, com maior parte das vítimas em idade reprodutiva, raça/cor negras, com local de ocorrência em via pública e por meio do uso de arma de fogo. Geralmente, as mulheres não parecem ser alvo de homicídio, a menos que sejam ameaçadas por alguém com quem têm vínculo ou, pelo menos, algum tipo de relacionamento anterior ${ }^{(11)}$.

Os homicídios intencionais de mulheres foram mais frequentes no grupo etário de 10 a 39 anos. Um estudo que analisou os homicídios de mulheres nas regiões do Brasil constatou que mulheres na segunda e terceira décadas de idade apresentaram maior risco de morte. $\mathrm{Na}$ Itália, Alemanha e Estados Unidos, os homicídios intencionais de mulheres ocorreram mais nas faixas de 15 a 29 anos e de 30 a 44 anos de idade ${ }^{(12)}$. Globalmente, esses grupos representam a maioria dos homicídios e mulheres com 30 anos ou mais podem ter maior risco de exposição à violência por parceiro íntimo ou familiar do que outros grupos etários ${ }^{(1)}$.

Em relação à raça/cor, observou-se que mais de $80 \%$ das mulheres vítimas de homicídios intencionais, em todas as RS, eram negras. Estudos têm demonstrado que a frequência do homicídio de mulheres varia de acordo com a raça/cor e, em geral, as mulheres negras são as principais vítimas. Nos Estados Unidos, a comparação das diferenças raciais e étnicas nos homicídios de mulheres adultas mostrou maior probabilidade de as mulheres negras serem mortas por conhecidos $(29,0 \%)^{(13)}$. Outros autores evidenciaram que a mortalidade por homicídios foi significativamente maior para mulheres negras $(\mathrm{OR}=2,1)$, quando comparadas com brancas $(\mathrm{OR}=1,0)$. Além disso, evidências indicaram que o tipo de violência que causou a morte das mulheres negras pode ter sido mais grave, como o uso de armas mais letais ou ferimentos cometidos em áreas do corpo que são mais vulneráveis ${ }^{(14)}$.

Neste estudo, a maior parte das mulheres era solteira, diferentemente do observado em outros estudos, nos quais as principais vítimas eram casadas ou encontravam-se em união estável e, em geral, o agressor é o parceiro íntimo ${ }^{(13,15)}$. A situação conjugal das vítimas na Declaração de Óbito está como solteira; entretanto, não há como saber, apenas com os dados do SIM, se essas mulheres tinham companheiros/namorados, para compatibilidade com a literatura, que mostra que elas são vitimadas por parceiros íntimos na maioria das vezes. Por meio do SIM não é possível responder se os homicídios de mulheres são provocados em razão do gênero. Porém, estima-se que cerca de $70 \%$ dessas mortes sejam feminicídios ${ }^{(16)}$.

No mundo, 13,5\% dos homicídios de mulheres são cometidos por um parceiro íntimo ${ }^{(3)}$. Relações hierárquicas de poder de gênero e transtornos decorrentes do uso de álcool e outras drogas podem estar relacionados ao comportamento violento ${ }^{(17)}$. O aumento da igualdade de gênero está associado a baixos níveis de violência, pois fornece às mulheres os recursos necessários para interromper um relacionamento insalubre ${ }^{(12)}$.
Um estudo sobre fatores associados ao homicídio por parceiro íntimo evidenciou que, nos casos nos quais o perpetrador tinha histórico de agressões, as vítimas foram 2,4 vezes mais propensas a sofrer a lesão fatal na sua residência. Igualmente, nos casos em que o autor era o atual parceiro da vítima, houve duas vezes mais chances de a lesão que ocasionou o óbito ocorrer na residência da vítima ${ }^{(18)}$. Na maioria das vezes, as vítimas estavam envolvidas em um episódio de agressão física ou verbal com um parceiro íntimo quando o homicídio aconteceu ${ }^{(18)}$.

O final de semana foi o período onde ocorreram mais homicídios. Tal fato pode estar relacionado ao aumento do consumo de bebidas alcoólicas nesse período. Um estudo realizado com vítimas de violência doméstica e familiar, atendidas em serviços de urgência e emergência, constatou que $46,1 \%$ dos casos ocorreram no final de semana ${ }^{(19)}$. Os meses de maior ocorrência de homicídios foram meses de férias e festas (carnaval, festas juninas e de fim de ano). Um estudo realizado no Brasil identificou que os meses de maior ocorrência de violência física contra mulheres foram setembro, outubro e dezembro ${ }^{(20)}$.

Houve predomínio dos homicídios por arma de fogo, e em três RS prevaleceram os homicídios por objeto cortante ou penetrante. Alguns estudos evidenciam que as armas de fogo, de diferentes tipos, são as mais envolvidas em homicídios de mulheres ${ }^{(13,21)}$. O uso de instrumentos afiados ou contundentes relaciona-se com o fato de o homicídio ter sido cometido em uma situação em que as facas, ou objetos semelhantes, podiam ser facilmente encontrados. Outros autores compreendem que instrumentos afiados, armas contundentes e outros métodos, como estrangulamento ou afogamento, podem representar um meio para agredir a vítima durante um conflito, enquanto que a arma de fogo indica a intenção de assassinar a vítima ${ }^{(22)}$.

Recente estudo de metanálise sobre os fatores de risco para os homicídios de mulheres por parceiros íntimos encontrou entre os principais fatores o fato de o agressor ter acesso direto a uma $\operatorname{arma}^{(23)}$. Parceiros íntimos violentos e que têm acesso a arma de fogo constituem-se autores de agressões mais graves. Nesse sentido, as legislações que proíbem que pessoas sob ordens de restrição devido à violência doméstica tenham acesso a essas armas estão associadas a reduções no número de homicídio por parceiro íntimo. Contudo, alguns dispositivos dessas leis e a sua aplicação podem afetar a sua eficiência ${ }^{(24)}$.

Com relação ao local de ocorrência dos homicídios, destaca-se a via pública. Ter sido este local o de maior ocorrência evidencia a intencionalidade e gravidade da violência, que acontece principalmente por meio de arma de fogo, o que dificulta a possibilidade de sobrevivência da vítima ${ }^{(25)}$.

Os maiores coeficientes de mortalidade e conglomerados críticos para homicídios de mulheres concentraram-se em municípios distantes da região metropolitana, localizados principalmente na Zona da Mata (III RS) e no Agreste pernambucano (IV RS). Fatores culturais podem influenciar nesse resultado, como a presença ainda forte dos padrões culturais de hierarquias de gênero. Ainda é frequente, em municípios localizados em regiões menos urbanas, as mulheres 
não denunciarem a violência doméstica ou estupros, principalmente em ambientes familiares ${ }^{(26)}$. Geralmente pelo medo do julgamento, que pode ser praticado pela sociedade local, medo de represálias e maior dificuldade de acesso a serviços de proteção às vítimas de violência, como as delegacias especializadas de atendimento à mulher ${ }^{(26)}$.

A análise espacial realizada neste estudo possibilitou tanto a identificação de áreas com aglomerados de município de alto risco para os homicídios de mulheres quanto áreas de transição epidemiológica, isto é, aquelas cercadas por municípios vizinhos de baixo risco. As áreas de transição podem ser consideradas de prioridade intermediária para as políticas públicas de prevenção da violência contra a mulher ${ }^{(9)}$. Um estudo que analisou espacialmente a distribuição das taxas de violência sexual praticada por parceiro íntimo contra mulheres mostrou que esse tipo de análise pode contribuir para a identificação de áreas vulneráveis e para a elaboração de estratégias de prevenção e fortalecimento de políticas públicas $^{(27)}$. De fato, a análise espacial dos homicídios é mais uma importante ferramenta a ser utilizada nas ações de prevenção da violência contra a mulher.

A quase totalidade dos municípios do estado de Pernambuco apresentou IDH entre médio e baixo, inclusive as áreas críticas para ocorrência de homicídios de mulheres. Quesitos como educação, saúde e renda podem interferir na violência de um local.

Em muitos casos os homicídios de mulheres são resultado de um ciclo de agressões. Os serviços de saúde têm papel fundamental na identificação e resposta às diversas situações de violência, pois muitas vezes são o primeiro local onde as vítimas buscam atendimento ${ }^{(21,28)}$. Tais serviços são responsáveis por acolher e implementar medidas preventivas e assistenciais e não apenas medicalização dos sinais e sintomas do adoecimento físico ${ }^{(29)}$. Torna-se necessário que os profissionais de saúde, inclusive da enfermagem, consigam reconhecer situações de violência contra a mulher. Também é preciso articular a notificação de violência contra a mulher com aconselhamento e a possibilidade de encaminhamento à rede composta pelos demais serviços de cuidado e proteção, visando a cessar o ciclo da violência ${ }^{(30)}$.

Esse estudo pode contribuir para ampliar os conhecimentos na área da saúde sobre os homicídios de mulheres, possibilitando uma maior visibilidade da violência contra as mulheres. Além disso, possibilitou a compreensão das características das mulheres que sofreram violência e morreram por consequência desta. Compreender as características das vítimas e dos crimes pode auxiliar a Enfermagem na tomada de decisões nas atividades preventivas e de assistência em situações de violência. Com a análise espacial foi possível identificar áreas críticas para ocorrência de homicídios de mulheres e estas podem ser áreas prioritárias para as intervenções na área da saúde pública.

Este estudo assume as seguintes limitações: (a) qualidade dos dados utilizados, embora seja registrada uma importante melhora do SIM; (b) dificuldades de acesso aos dados da segurança pública, que contém informações sobre as circunstâncias, a relação da vítima com o agressor e o uso de substâncias psicoativas, pois essas informações possibilitariam uma abordagem metodológica estruturada; e (c) parcela significativa dos homicídios de mulheres são, possivelmente, feminicídios; todavia, o SIM não contém essa informação. Estudos epidemiológicos futuros devem fundamentar-se numa análise metodológica que inclua múltiplas bases de dados (Sistema de Informação de Agravos de Notificação Sinan, Serviço de Atendimento Móvel de Urgência - Samu, segurança pública) e fatores associados aos homicídios, além de considerar a contextualização dos comportamentos e condutas que possam ser preditivas de homicídios.

\section{CONCLUSÃO}

A redução da violência letal contra as mulheres requer medidas abrangentes que abordem fatores individuais, sociais, econômicos, culturais e situacionais. Neste estudo evidencia-se que mulheres jovens, negras e solteiras foram as maiores vítimas. Os homicídios ocorreram em via pública e por meio do uso de arma de fogo. Os óbitos aconteceram no final de semana e nos meses de férias e festas tradicionais no Brasil (carnaval, festas juninas e de fim de ano). Os maiores coeficientes de mortalidade e áreas críticas concentraram-se em municípios distantes da região metropolitana, localizados principalmente na Zona da Mata e no Agreste pernambucano.

A caracterização dos homicídios de mulheres pode contribuir para a ampliação dos conhecimentos sobre violência contra as mulheres na área da enfermagem e da saúde pública e podem ser utilizados na condução de medidas que visam à redução dos homicídios. Com a análise espacial foi possível identificar áreas críticas para ocorrência de homicídios e estas podem ser áreas prioritárias para as intervenções na área da saúde.

\section{RESUMO}

Objetivo: Analisar as características e a distribuição espacial dos homicídios intencionais de mulheres, de acordo com as regionais de saúde, em um estado da Região Nordeste do Brasil. Método: Estudo ecológico que teve o município e as regiões de saúde como unidades de análise e usou dados do Sistema de Informações sobre Mortalidade. Calcularam-se as taxas de mortalidade, que foram suavizadas pelo método bayesiano empírico local. Para identificação da existência de autocorrelação espacial, utilizou-se o índice de Moran. Resultados: Registraram-se 1.144 homicídios, com taxa média de 6,2/100.000 mulheres. Para todas as regiões de saúde prevaleceram a faixa etária de 10 a 39 anos, a raça/cor negra e a situação conjugal solteira. Os homicídios ocorreram em via pública. Os dias da semana com maiores registros foram sábado e domingo e os meses do ano dezembro, janeiro, fevereiro e junho. O índice de Moran global foi de 0,6 ( $\mathrm{p}=0,01)$. Identificaram-se clusters de municípios com altas taxas de homicídios nas III e IV regiões de saúde. Conclusões: As vítimas de homicídios eram jovens, negras e solteiras. A análise espacial localizou áreas críticas de ocorrência de homicídios, que são prioritárias para as ações de prevenção da violência contra as mulheres.

Homicídio; Violência contra a Mulher; Sistemas de Informação; Estatísticas Vitais; Análise Espacial. 


\section{RESUMEN}

Objetivo: Analizar las características y la distribución espacial de los homicidios intencionales de mujeres, de acuerdo con las regionales de salud, en una provincia de la Región Noreste de Brasil. Método: Estudio ecológico que utilizó el municipio y las regiones de salud como unidades de análisis y empleó datos del Sistema de Informaciones sobre Mortalidad. Se calcularon las tasas de mortalidad que fueron mitigadas por el método bayesiano empírico local. Se utilizó el índice de Moran para identificación de la existencia de autocorrelación espacial. Resultados: Fueron registrados 1.144 homicidios con un promedio de 6,2/100.000 mujeres. En todas las regiones de salud predominaron mujeres con edades entre 10 y 39 años, de raza/color negro y solteras. Los homicidios ocurrieran en vía pública. Los días en los que más ocurrieron fueron sábado y domingo y los meses diciembre, enero, febrero y junio. El índice de Moran global fue 0,6 ( $p=0,01)$. Se identificaron clusters de municipios con altas tasas de homicidios en las III y IV regiones de salud. Consideraciones Finales: Las victimas de homicidios eran jóvenes, negras y solteras. El análisis espacial localizó áreas críticas de ocurrencia de homicidios, que son prioritarias para las acciones preventivas de la violencia contra las mujeres.

\section{DESCRIPTORES}

Homicidio; Violencia contra la Mujer; :Sistemas de Información; Estadísticas Vitales; Análisis Espacial.

\section{REFERÊNCIAS}

1. United Nations. Office on Drugs and Crime. Global study on homicide 2013. [Internet]. New York: United Nations; 2014 [cited 2019 Jan 14]. Available from: http://www.unodc.org/gsh/

2. Sheehan BE, Murphy SB, Moynihan MM, Dudley-Fennessey E, Stapleton JG. Intimate partner homicide: new insights for understanding lethality and risks. Violence Against Women. 2015;21(2):269-88. https://doi.org/10.1177/1077801214564687

3. Stöckl H, Devries K, Rotstein A, Abrahams N, Campbell J, Watts C, et al. The global prevalence of intimate partner homicide: a systematic review. Lancet. 2013;382(9895):859-65. https://doi.org/10.1016/S0140-6736(13)61030-2

4. Caman S, Kristiansson M, Granath S, Sturup J. Trends in rates and characteristics of intimate partner homicides between 1990 and 2013. J Crim Justice. 2017;49:14-21. https://doi.org/10.1016/j.jcrimjus.2017.01.002.

5. Sanz-Barbero B, Vives-Cases C, Otero-García L, Muntaner C, Torrubiano-Domínguez J, O'Campo P. Intimate partner violence among women in Spain: the impact of regional-level male unemployment and income inequality. Eur J Public Health. 2015;25(6):1105-11. https://doi.org/10.1093/eurpub/ckv048

6. Sandoval GA, Marinho F, Delaney R, Pinto IV, Lima CM, Costa RM, et al. Mortality risk among women exposed to violence in Brazil: a population-based exploratory analysis. Public Health. 2020;179:45-50. https://doi.org/10.1016/j.puhe.2019.09.019

7. Souza ER, Meira KC, Ribeiro AP, Santos JD, Guimarães RM, Borges LF, et al. Homicides among women in the different Brazilian regions in the last 35 years: an analysis of age-period-birth cohort effects. Cien Saúde Colet. 2017;22(9):2949-62. https://doi.org/10.1590/1413-81232017229.12392017

8. Instituto de Pesquisa Econômica Aplicada. Atlas da Violência 2020 [Internte]. Brasília, DF: Instituto de Pesquisa Econômica Aplicada; 2020 [cited 2020 June 27]. Available from: https://www.ipea.gov.br/portal/index.php?option=com_content\&id=33410

9. Oliveira AL, Luna CF, Quinino LR, Magalhães MA, Santana VC. Spatial analysis of intentional homicides in João Pessoa, Paraíba, Brazil, 2011-2016. Epidemiol Serv Saúde. 2019;28(1):e2018184. https://doi.org/10.5123/S1679-49742019000100003

10. Brasil. Programa das Nações Unidas para o Desenvolvimento. Desenvolvimento humano e IDH [Internet]. Brasília, DF: Programa das Nações Unidas para o Desenvolvimento; 2020 [cited 2020 June 10]. Available from: https://www.br.undp.org/content/brazil/pt/home/idh0.html

11. Iratzoqui A, McCutcheon J. The influence of domestic violence in homicide cases. Homicide Stud. 2018;22(2):145-60. https://doi.org/10.1177/1088767917751673.

12. Terranova C, Zen M. Women victims of intentional homicide in Italy: new insights comparing Italian trends to German and U.S. trends, 2008-2014. J Forensic Leg Med. 2018;53:73-8. https://doi.org/10.1016/j.jflm.2017.11.007

13. Petrosky E, Blair JM, Betz CJ, Fowler KA, Jack SP, Lyons BH. Racial and Ethnic differences in homicides of adult women and the role of intimate partner violence - United States, 2003-2014. MMWR Morb Mortal Wkly Rep. 2017;66(28):741-6. https://doi.org/10.15585/mmwr.mm6628a1

14. Gonzalez-Guarda RM, Luke B. Contemporary homicide risks among women of reproductive age. Women's Health Issues. 2009;19(2):119-25. https://doi.org/10.1016/j.whi.2009.01.002

15. Moreschi C, Da Broi U, Zamai V, Palese F. Medico legal and epidemiological aspects of femicide in a judicial district of north eastern Italy]. J Forensic Leg Med. 2016;39:65-73. https://doi.org/10.1016/j.jflm.2016.01.017

16. Margarites AF, Meneghel SN, Ceccon RF. Feminicides in Porto Alegre: How many? Who are they?. Rev Bras Epidemiol. 2017;20(2):225-36. https://doi.org/10.1590/1980-5497201700020004

17. Gattegno MV, Wilkins JD, Evans DP. The relationship between the Maria da Penha Law and intimate partner violence in two Brazilian states. Int J Equity Health. 2016;15(1):138. https://doi.org/10.1186/s12939-016-0428-3

18. Yousuf S, McLone S, Mason M, Snow L, Gall C, Sheehan K. Factors associated with intimate partner homicide in Illinois, 2005-2010: findings from the Illinois Violent Death Reporting System. J Trauma Acute Care Surg. 2017;83(5S Suppl 2):S217-21. https://doi.org/10.1097/TA.0000000000001578

19. Garcia LP, Duarte EC, Freitas LR, Silva GD, Garcia LP, Duarte EC, et al. [Domestic and family violence against women: a case-control study with victims treated in emergency rooms]. Cad Saude Publica. 2016;32(4):e00011415. Portuguese. https://doi.org/10.1590/0102-311X00011415

20. Cavalcante GM, Bernardino IM, Nóbrega LM, Ferreira RC, Ferreira E, d'Avila S. Temporal trends in physical violence, gender differences and spatial vulnerability of the location of victim's residences. Spat Spatio-Temporal Epidemiol. 2018;25:49-56. https://doi.org/10.1016/j.sste.2018.02.001

21. García-Moreno C, Hegarty K, d'Oliveira AF, Koziol-McLain J, Colombini M, Feder G. The health-systems response to violence against women. Lancet. 2015;385(9977):1567-79. https://doi.org/10.1016/S0140-6736(14)61837-7 
22. Carmichael H, Jamison E, Bol KA, McIntyre R Jr, Velopulos CG. Premeditated versus "passionate": patterns of homicide related to intimate partner violence. J Surg Res. 2018;230:87-93. https://doi.org/10.1016/j.jss.2018.04.050

23. Spencer CM, Stith SM. Risk factors for male perpetration and female victimization of intimate partner homicide: a meta-analysis. Trauma, Violence, Abus. 2020;21(3):527-40. https://doi.org/10.1177/1524838018781101

24. Zeoli AM, Malinski R, Turchan B. Risks and targeted interventions: firearms in intimate partner violence. Epidemiol Rev. 2016;38(1):125-39. https://doi.org/10.1093/epirev/mxv007

25. Trindade RF, Costa FA, Silva PP, Caminiti GB, Santos CB. Map of homicides by firearms: profile of the victims and the assaults. Rev Esc Enferm USP. 2015;49(5):748-55. https://doi.org/10.1590/S0080-623420150000500006

26. Borth LC, Costa MC, Silva EB, Fontana DG, Arboit J. Network to combat violence against rural women: articulation and communication of services. Rev Bras Enferm. 2018;71 suppl 3:1212-9. https://doi.org/10.1590/0034-7167-2017-0044

27. Fontes KB, Alarcão ACJ, Nihei OK, Pelloso SM, Andrade L, Carvalho MDB. Regional disparities in the intimate partner sexual violence rate against women in Paraná State, Brazil, 2009-2014: an ecological study. BMJ Open. 2018;8(2):e018437. https://doi.org/10.1136/bmjopen-2017-018437

28. Garcia LP, Garcia LP. The invisible magnitude of violence against women. Epidemiol Serv Saude. 2016;25(3):451-4. https://doi.org/10.5123/S1679-49742016000300001

29. Oliveira RN, Fonseca RM. Health needs: the interface between the discourse of health professionals and victimized women. Rev Lat Am Enfermagem. 2015;23(2):299-306. https://doi.org/10.1590/0104-1169.3455.2555

30. Barufaldi LA, Souto RM, Correia RS, Montenegro MM, Pinto IV, Silva MM, et al. Gender violence: a comparison of mortality from aggression against women who have and have not previously reported violence. Cien Saude Colet. 2017;22(9):2929-38. https://doi.org/10.1590/141381232017229.12712017

\section{Apoio financeiro}

Coordenação de Aperfeiçoamento de Pessoal de Nível Superior - Brasil (Capes). Código de Financiamento 001. 